\title{
The Role of Self-compassion, Concern for Others, and Basic Psychological Needs in the Reduction of Caregiving Burnout
}

\author{
Zach Gerber $^{1,2} \cdot$ David Anaki ${ }^{1,3}$ (B) \\ Accepted: 27 October 2020 / Published online: 16 November 2020 \\ (C) Springer Science+Business Media, LLC, part of Springer Nature 2020
}

\begin{abstract}
Objectives Compassion is the beneficial response of an individual to the sufferings and difficulties of others. Self-compassion refers to feelings of concern an individual feel towards oneself. Both may serve as potential buffers in a hospital setting where caregivers attending to patients in critical condition experience emotional distress, which may lead to burnout. However, the unique contribution of compassion, both to self and others, to the decrease of burnout is unclear.

Methods In the present study, we recruited 109 professional caregivers working in a hospital's intensive care and rehabilitative units. We explored a theoretically driven mediation model in which the relationship between compassion for self and others and burnout is mediated by different basic psychological needs, detailed in the self-determination theory.

Results Results indicated that self-compassion and concern for others were associated with reduced levels of burnout. Each of these buffers against burnout was mediated by a unique psychological need, thereby supporting a three-layered model of (a) compassion and self-compassion, (b) basic psychological needs, and (c) burnout.

Conclusions Our study highlights compassion and self-compassion as potential resilience factors against the challenge of burnout in healthcare. It points to promising avenues for preemptive clinical interventions.
\end{abstract}

Keywords Self-compassion $\cdot$ Concern for others $\cdot$ Caregiving $\cdot$ Burnout $\cdot$ Self-determination theory

Compassion, or empathic concern for others, refers to the positive emotions one feels towards others' welfare coupled with the motivation to help alleviate their sufferings and pains (Strauss et al. 2016). Beyond feeling empathic concern for a person who is suffering, the concept of compassion consists of the motivation to relieve him from his sufferings (Goetz et al. 2010; Lazarus 1991). In addition to these affective and motivational aspects of compassion, others added a third cognitive facet-noticing. Noticing is acknowledging the existence of misery in an individual (Kanov et al. 2004). Gilbert (2009) noted that compassion involves additional attributes, such as being non-judgmental towards others and tolerating personal

David Anaki

david.anaki@biu.ac.il

1 Department of Psychology, Bar-Ilan University, 52900 Ramat-Gan, Israel

2 Reuth Rehabilitation Hospital, 61092 Tel Aviv, Israel

3 The Leslie and Susan Gonda (Goldschmied) Multidisciplinary Brain Research Center, Bar-Ilan University, Bldg. 901, Room 202, 52900 Ramat-Gan, Israel distress when faced with other people's suffering. Thus, compassion is a multi-faceted construct, comprised of various elements (Khoury 2019; Woodruff and Stevens 2018).

Compassion has commonalities with other related concepts, such as kindness, pity, and empathy (Strauss et al. 2016). One of these related concepts is self-compassion, which denotes a condition in which compassion is felt towards oneself. Self-compassion, according to one eminent view (Neff 2003a, b), entails three main components that overlap and mutually interact: self-kindness (with its self-judgment counterpart), feelings of common humanity (versus isolation), and mindfulness (in contrast to over-identification). Selfkindness is the tendency to be caring and understanding of oneself rather than harshly critical. When experiencing difficulty, self-compassionate people embrace themselves, providing self-comfort rather than self-persecution. The second component, common humanity, is the recognition that all people fail, err, and experience pain. The self-compassionate individual perceives difficulty and failure as an inevitable part of shared human experience, enabling one to feel the proximity to others when encountering challenges. Mindfulness, the third component of self-compassion, refers to the awareness 
of the present moment's experience in a clear and balanced manner. Thus, the person neither ignores nor ruminates on troubling aspects of oneself or one's environment. Mindfulness involves taking a broad perspective regarding lived experience beyond transient pain (Brown and Ryan 2003). Self-compassion has been associated with reduced negative emotions, such as self-criticism, depression, anxiety, rumination, thought suppression, perfectionism, and eating disorders (Neff et al. 2018). Of particular relevance to the current study is the association between self-compassion and less avoidant coping patterns, reduced PTSD following exposure to traumatic events, and reduced burnout among mental health and primary healthcare workers (Finlay-Jones et al. 2015; Montero-Marin et al. 2016; Walker 2017; Ying and Han 2009). Recently, Neff et al. (2020) demonstrated how self-compassion cultivation among pediatric caregivers reduced their burnout levels.

The similarities between compassion and self-compassion have led to a discussion of how these two concepts overlap. Some researchers focused on the conceptual linkages between self-compassion and compassion and claimed that the two emotions overlap, differing only in the object they direct to (e.g., Gilbert 2009; Strauss et al. 2016). Others stressed the importance of empirical research findings (e.g., Pommier et al. 2020). Explorations of the relationship between selfcompassion and concern for others in different populations revealed, thus far, no significant correlations or weak ones at best (e.g., Neff and Pommier 2013). Mills et al. (2018) found a weak negative correlation between the two concepts among palliative caregivers. Gerber et al. (2015) also showed that the two concepts did not share any covariance and that they reflected different psychological self-needs, considered essential components in self-determination theory (Deci et al. 2017; Ryan and Deci 2000, 2017). Specifically, self-compassion expressed a sense of autonomy and choice, while concern for others reflected a feeling of communion and relatedness.

Self-determination theory (SDT) is a macro-theory of human motivation applied to various domains, including that of the workplace (Deci et al. 2017; Ryan and Deci 2000, 2017). The theory differentiates between two types of motivations, intrinsic and extrinsic. Intrinsic motivation consists of acting according to personal values and interests while experiencing a sense of choice, initiation, and endorsement of one's behavior. Conversely, extrinsic motivation involves behaving with a sense of coercion, pressure, and contingency (Ryan \& Deci 2000). Accordingly, the type of motivation that underlies individuals' behavior will determine their psychological wellbeing and performance. Furthermore, SDT underscores the crucial role of basic psychological needs satisfaction in promoting mental health, wellbeing, and enhanced performance (Ryan and Deci 2017). These needs, also referred to as psychological nutriments, consist of autonomy, competence, and relatedness. Autonomy refers to the sense of self- causality and the feeling that one is engaged in an activity stemming from his independent will and choice. Competence is feeling effective in one's interactions with the natural and social environment. Finally, relatedness is the need to feel connected and involved with others and experience a sense of belonging. These nutriments may be experienced independently, yet they are all essential for sustaining intrinsic motivation.

Being a macro-theory of motivation in social contexts, a primary focus of SDT is motivation in the workplace, with broad consequences regarding the interplay between employers and employees in organizational settings (Gagné and Deci 2005, 2014). One such implication is the essentiality of organizational support for intrinsic motivation and satisfaction of the employees' three psychological needs in preventing occupational burnout. Occupational burnout, a major detrimental factor to individuals' wellbeing and job performance, results from unsuccessful chronic work-related stress management. It is considered a pressing issue among workers in the health professions in particular (International Statistical Classification of Diseases and Related Health Problems, 11th revision; ICD-11). Several components characterize burnout. Emotional exhaustion, described as feelings of being emotionally drained by one's work, is the central dimension of burnout. Cynicism is a negative or indifferent response to the tasks at work and the individuals they interact with while performing their job. Finally, depletion of personal accomplishment refers to a decline in one's feelings of competence and achievement at work overtime (Maslach et al. 1997; Schaufeli et al. 2009). Health care personnel working in critical care settings are highly vulnerable to severe occupational stress and, consequently, burnout due to patients' death and loss firsthand as well as the grief of their family members, and encountering the physical consequences of abuse, neglect, and violence (Moss et al. 2016). Psychological need satisfaction has been put forth as an effective framework in counteracting the development of burnout. Indeed, several studies have examined the contribution of autonomous motivation and psychological need satisfaction in reducing occupational burnout (Bakker and Costa 2014; Deci et al. 2017; Deci and Ryan 2014). Their findings underscored the importance of need satisfaction as a buffer against burnout and their role in establishing occupational wellbeing and performance. Focusing on the specific relationship between need satisfaction and burnout in the health care professions, Trépanier et al. (2013) found among nurses that need-thwarting behaviors, such as bullying, reduced work engagement, and increased burnout.

The socio-psychological conditions that underlie intrinsic motivation and psychological needs have been elaborately examined. However, research has mostly overlooked the psychological processes that influence specific individual psychological needs and motivations (but see Ronen and 
Mikulincer 2014). Indeed, individual differences are essential factors that appear in models of SDT applications in the workplace (e.g., Deci et al. 2017). However, these models highlight individual orientations that are mainly work-related, such as aspirations, goals, and causality orientations. Researchers have paid less attention to the role of personality traits and feelings or attitudes toward life as critical factors in the SDT paradigm (but see Sheldon and Prentice 2019).

Self-compassion is a coping strategy that helps individuals face their fate and the consequence of their deeds (Gerber and Anaki 2019). It provides the capacity to take responsibility for one's actions without resorting to defense mechanisms that distort or deny it (Allen and Leary 2010). As a result, the individual is empowered to make wise decisions and select optimal responses. Therefore, self-compassion enables individuals to create a direct affinity between themselves and their behavior, legitimizing their actions and inactions beyond their mere consequences. Autonomy also refers to individuals' feelings that their activities stem from their own volitions (selfcausality, Deci and Ryan 2014). A sense of autonomy enables people to feel responsible for their actions without alienating themselves from them (Ryan et al. 2012). This interpretation of autonomy converges with the premise of self-compassion and presumably is facilitated and maintained by it (Legault and Inzlicht 2013). Consequently, enhanced autonomy improves performance by accepting negative affect and openness to threatening self-relevant information when encountering difficulty and limitations (Hodgins et al. 2010; Hodgins and Knee 2002;).

Furthermore, concern for others reflects awareness and empathy for others' needs or sufferings, coupled with experiencing motivation to respond and engage wherever possible. An important distinction has been made between "pathological" concern, which favors the care of others over one's own self needs and "healthy" concern, where the caring treatment of others is a form of genuine self-expression that naturally develops when conditions permit (Tolmacz 2010). Findings indicate that healthy concern reflects secure attachment, reduced psychopathology, and a better self-perception, all in contrast to pathological concern (Gerber et al. 2015). Thus, healthy concern may fulfill the need for relatedness, essential for establishing wellbeing in daily life and work (Deci and Ryan 2014). Therefore, caregivers who experience healthy concern for others will nourish their need for relatedness in their daily work. We note that it is hard to determine the directionality between these two concepts. On the one hand, concern for others appears to be a prerequisite for fulfilling the nutriment of relatedness - an individual who cares for others will ultimately feel an authentic sense of belonging when doing so. On the other hand, concern for others may arise as a result of the need for relatedness. In our theoretical model, though, we adopt the former possibility since SDT research has indicated that relatedness serves as a direct buffer against burnout (e.g.,
Trépanier et al. 2013). In light of this finding, relatedness is conceptually better suited to serve as a mediator between concern for others and burnout.

In our model, while both autonomy and relatedness mediate the relationship between self-compassion and concern for others, respectively, and burnout, the self-need of competence does not. Self-compassion does not appear to augment competence, but rather it may alleviate the inner response to personal incompetence. Furthermore, concern for others does not seem to be necessarily related in a unique way to competence. Notably, in the Gerber et al. (2015) study, competence was associated with self-compassion and concern for others, indicating that competence does not address their lack of covariance. In the present research, we presumed that selfcompassion and concern for others would relate to occupational burnout, among caregivers, through different psychological needs. We hypothesized that the covariance between self-compassion and burnout will be meditated by autonomy and that the covariance between concern for others and burnout will be meditated by relatedness.

\section{Method}

\section{Participants}

Participants were 109 professional caregivers (81\% woman) working in rehabilitation and critical care units in a major rehabilitation hospital. The sample size (i.e., 109) was determined by an a priori power analysis for linear multiple regression (fixed model, $R^{2}$ increase), with an alpha of 0.05 , power of 0.80 , a medium-size effect $\left(f^{2}=.15\right)$, and eight predictors (G*Power, Faul et al. 2007). The mean age of the participants was 39.7 (range $25-67, \mathrm{SD}=12.7$ ). The majority of the sample was either married $(63 \%)$ or cohabitating $(10 \%)$, with the remaining participants either divorced (12\%) or single (12\%). Following a recent investigation of burnout among oncology nurses (Potter et al. 2010), we also collected data regarding the caregivers' general care experience in the healthcare system (GCE) and their particular clinical experience at the current rehabilitation hospital (PCE, both in years). For the distribution of professional affiliation, age, and clinical experience of the participants, see Table 1.

\section{Procedures}

Following approval from the ethics committees of Reuth Rehabilitation Hospital (Tel Aviv, Israel) and of the Psychology Department at Bar-Ilan University (Ramat-Gan, Israel), the first author approached various staff members during work hours, distributed the questionnaires, and requested their participation. We did not offer any compensation in exchange for taking part in the study. All consenting participants 
Table 1 Professional affiliation and experience (GCE and PCE) of the caregivers' sample

\begin{tabular}{|c|c|c|c|c|c|}
\hline & $\begin{array}{l}\text { Frequency } \\
(N)\end{array}$ & $\begin{array}{l}\text { Mean age } \\
\text { (years) }\end{array}$ & $\begin{array}{l}\text { Mean GCE } \\
\text { (years) }\end{array}$ & $\begin{array}{l}\text { Mean PCE } \\
\text { (years) }\end{array}$ & $\begin{array}{l}\text { Gender } \\
\text { (\% male) }\end{array}$ \\
\hline \multicolumn{6}{|l|}{ Professional affiliation } \\
\hline Medicine & 8 & $42(9.30)$ & $13.25(12.62)$ & $7.63(7.57)$ & 37 \\
\hline Nursing & 39 & $46(10.77)$ & $18.22(11.32)$ & $13.43(10.70)$ & 19 \\
\hline Physical therapy & 19 & $36(8.85)$ & $8.74(7.80)$ & $6.74(4.61)$ & 21 \\
\hline Occupational therapy & 14 & $32(7.72)$ & $6.64(6.78)$ & $6.21(6.14)$ & 7 \\
\hline Speech therapy & 2 & $41(3.54)$ & $12(5.65)$ & $12(5.65)$ & 0 \\
\hline Nutrition & 5 & $36(4.66)$ & $8(4.63)$ & $7.80(4.76)$ & 0 \\
\hline Psychosocial & 21 & $40(12.49)$ & $9.55(9.57)$ & $5.79(8.41)$ & 10 \\
\hline
\end{tabular}

$G C E$, general care experience; $P C E$, particular clinical experience. SDs in parentheses

filled out the four questionnaires during their available time between March and October 2017. Out of 150 questionnaires distributed, 109 were returned, approximating a $70 \%$ response rate. The participants were debriefed following the completion and return of the questionnaires to the first author.

\section{Measures}

Self-Compassion We utilized the 26-item Self-Compassion Scale (SCS, Neff 2003a). The scale assesses six different aspects of self-compassion (with negative aspects reverse-coded): self-kindness (e.g., "I try to be understanding and patient toward aspects of my personality I don't like"), self-judgment (e.g., 'I'm disapproving and judgmental about my own flaws and inadequacies"), common humanity (e.g., "I try to see my failings as part of the human condition"), isolation (e.g., "When I think about my inadequacies it tends to make me feel more separate and cut off from the rest of the world"), mindfulness (e.g., "When something painful happens I try to take a balanced view of the situation"), and over-identification (e.g., "When I'm feeling down I tend to obsess and fixate on everything that's wrong"). Responses are given on a five-point scale from almost never to almost always (Neff 2003a). The internal consistency of the SCS in the present study was 0.83 . In the current study, we computed an overall SCS average for each participant. Although some have pleaded against using the total score (Muris et al. 2016; Muris and Petrocchi 2016), others have claimed that the factor structure of the SCS supports the use of a total score (Neff et al. 2019).

Concern for others We utilized part of the Interpersonal Reactivity Index, which has been conceptualized as the proper measurement of healthy concern (IRI, Davis 1980; Tolmacz 2010). We used the Empathic Concern subscale, an eight-item scale, which evaluates the degree to which the respondent feels warmth, compassion, and concern toward others (e.g., "Seeing warm emotional scenes melts my heart and makes me teary-eyed"). This subscale assesses healthy concern as an emotional component only. Participants rate the extent to which each item was descriptive of their feelings on a fivepoint scale ranging from very not me (1) to very me (5). Cronbach's alpha was 0.79 .

Psychological Needs (PNS, Deci and Ryan 2000) The scale includes separate subscales measuring the satisfaction levels of needs for autonomy ("I feel like I can pretty much be myself in my daily situations"), competence ("Most days I feel a sense of accomplishment from what I do"), and relatedness in life ("I consider the people I regularly interact with to be my friends"). The reliability of the scale in the study was 0.78 . We believe that psychological needs' satisfaction of caregivers is expressed across all contexts, personal as well as occupational. Therefore, we used the more general 21-item Basic Psychological Needs Scale instead of the basic needs in the workplace context.

Burnout As a burnout measure, we administered the Maslach Burnout Inventory (MBI, Maslach et al. 1997). The MBI is comprised of three dimensions of burnout (a) emotional exhaustion, characterized as depletion of emotional resources, fatigue, and lack of energy (e.g., "I feel like I'm at the end of my rope"), (b) depersonalization, described as a loss of concern and compassion towards clients resulting in inappropriate behavior (e.g., "I worry that my work is hardening me emotionally"), and (c) a diminished sense of personal accomplishment (e.g., "I have accomplished many worthwhile things in this job"). The scale has 22 items that are answered on a six-point Likert scale and indexes the cumulative effects of work-related stressors (Maslach 1982). Cronbach's alpha coefficient was 0.85 .

\section{Data Analyses}

We calculated participant demographic characteristics, zeroorder correlations, and hierarchical regression with the SPSS 
22 software. To examine our hypotheses, we used a structural equation modeling approach, which is a method to test the fit of a hypothesized model with empirical data. Because of our limited data set, the Partial Least Squares (PLS) SEM path modeling approach was selected (Ringle et al. 2015).

\section{Results}

Data were examined for normality, multicollinearity, completeness, and the presence of outliers or impossible values. Missing values were minimal $(\sim 2 \%)$ and were either replaced with the variable mean or pairwise deleted. To estimate the unmediated relations among the variables of interest and demographic variables, we conducted a zero-sum correlation analysis (Table 2). Notably, self-compassion and concern for others were unrelated $(r=.09, p>.39)$. The three psychological needs were inter-correlated, with correlations ranging from .31 to .49 ( $p$ 's $<.01)$. Self-compassion was correlated with autonomy $(r=.45)$ and competence $(r=.40)$, but not with relatedness $(r=.14)$. On the other hand, concern for others was correlated with relatedness $(r=.36)$ and competence $(r=.20)$, but not with autonomy $(r=.10)$. Burnout was negatively correlated with both concern for others $(r=-.25$, $p>.05)$ and self-compassion $(r=-.34, p>.01)$. Additionally, burnout was associated with all the psychological needs (range $=-.40$ to $-.50, p$ 's $<.01)$. Finally, burnout was significantly associated with age $(r=-.22)$, but not with the two measures of experience (i.e., GCE and PCE), which were highly correlated with each other $(r=.80)$.

To determine the unique contribution of self-compassion and concern for others to participants' burnout, we conducted a three-step hierarchical regression analyses. In the first step of the analysis, we entered participants' age and measures of clinical experience (GCE, PCE). In the second step, we added self-compassion and concern for others as predictors to determine their unique contribution to burnout beyond the contribution of age and years of experience. Finally, in the third step, we added the psychological need measures of autonomy, relatedness, and competence as predictors. This third step allowed us to examine the role of self-needs to burnout beyond the previous factors' contribution.

The first step was significant, $F(3,105)=2.83, p<.05$, $R^{2}=.08$, with age making a significant contribution $(\beta=$ $-.36, p<.05$, Table 3 ). The second step in the regression also yielded a significant change in the variance accounted for, $\Delta F(2,103)=9.10, p<.001, \Delta R^{2}=.14$, with self-compassion and concern for others making a significant contribution $(\beta=$ $-.31, p<.001$, and $\beta=-.18, p<.05$, respectively). In the third step, the self-needs scores' addition yielded a significant change in the variance accounted for, $\Delta F(3,100)=10.21, p>.001$, $\Delta R^{2}=.18$, with autonomy and relatedness making a significant contribution $(\beta=-.29, p<.05$, and $\beta=-.25, p<.05$, respectively), as opposed to competence, which did not $(\beta=-.11$, $p=23$ ). In addition, self-compassion and concern for others were no longer significantly associated with burnout after the self-needs scores were added to the predictors set. PCE and age were, however, significant.

The path model's goodness of fit was assessed by measuring the standardized root mean square residual (SRMR, Henseler et al. 2016).). The SRMR value of 0.086 confirmed the overall model fit (Garson 2016). The insample predictive power of the model was assessed using the coefficient of determination $\left(R^{2}\right)$. The $R^{2}$ of behavior was equal to 0.40 . The out-of-sample predictive value was evaluated using the blindfolding procedure (omission distance $=7$ ). A $Q^{2}$ value above zero in the cross-validated redundancy report confirms predictive relevance. All $Q^{2}$ values were significantly greater than zero. The effect sizes $\left(f^{2}\right)$ of self-compassion on autonomy and competence were medium-high ( 0.25 and 0.18 , respectively). The effect sizes of concern on relatedness and competence were 0.11 and 0.03 , respectively. Finally, the effects of

Table 2 Zero-sum correlations between all variables

\begin{tabular}{|c|c|c|c|c|c|c|c|c|c|c|}
\hline Gender & $\begin{array}{l}\text { Gender } \\
1\end{array}$ & Age & GCE & PCE & $\mathrm{SC}$ & Concern & Autonomy & Competence & Relatedness & Burnout \\
\hline Age & .00 & 1 & & & & & & & & \\
\hline GCE & .09 & $.80 * *$ & 1 & & & & & & & \\
\hline PCE & .01 & $.66^{* *}$ & $.80 * *$ & 1 & & & & & & \\
\hline Self-compassion & -.16 & .12 & .17 & .12 & 1 & & & & & . \\
\hline Concern for others & $-.22 *$ & .11 & .00 & .00 & .09 & 1 & & & & \\
\hline Autonomy & .14 & $.25 *$ & .19 & .15 & $.45^{* *}$ & .10 & 1 & & & \\
\hline Competence & .05 & .07 & .07 & .04 & $.40 * *$ & $.20 *$ & $.49 * *$ & 1 & & \\
\hline Relatedness & .14 & .03 & .04 & .13 & .14 & $.36 * *$ & $.31 * *$ & $.34 * *$ & 1 & \\
\hline Burnout & .03 & $-.22 *$ & -.11 & -.01 & $-.34 * *$ & $-.25 *$ & $-.50 * *$ & $-.40 * *$ & $-.42 * *$ & 1 \\
\hline
\end{tabular}

$G C E$, general care experience; $P C E$, particular clinical experience; $S C$, self-compassion. $* p<.05, * * p<.01$ 
Table 3 (Un)standardized regression coefficients predicting burnout

\begin{tabular}{|c|c|c|c|}
\hline & \multicolumn{2}{|c|}{ Burnout } \\
\hline & Variable & $B$ & $\beta$ \\
\hline \multirow[t]{3}{*}{ Step 1} & Age & -.50 & $-.36^{*}$ \\
\hline & GCE & .01 & .01 \\
\hline & PCE & .38 & .22 \\
\hline \multirow[t]{5}{*}{ Step 2} & Age & -.45 & $-.32 *$ \\
\hline & GCE & .05 & .03 \\
\hline & PCE & .37 & .21 \\
\hline & Self-Compassion & -9.54 & $-.31 * *$ \\
\hline & Concern for Others & -5.45 & $-.18^{*}$ \\
\hline \multirow[t]{8}{*}{ Step 3} & Age & -.37 & $-.27 *$ \\
\hline & GCE & -.02 & -.02 \\
\hline & PCE & .46 & $.27 *$ \\
\hline & Self-Compassion` & -3.77 & -.12 \\
\hline & Concern for Others & -2.26 & -.08 \\
\hline & Autonomy & -6.18 & $-.29 * *$ \\
\hline & Competence & -1.90 & -.11 \\
\hline & Relatedness & -5.41 & $-.25 * *$ \\
\hline
\end{tabular}

$* p<.05, * * p<.01$

autonomy and relatedness on burnout were both 0.08 . All other effect sizes were smaller than 0.02 .

The analysis revealed that self-compassion indirectly influenced burnout through its effect on autonomy. As can be seen in Fig. 1, participants with higher selfcompassion scores had higher autonomy needs than participants with lower self-compassion scores (path $a$ coefficient $=0.45, p<.001$ ), and participants who had high autonomy needs reported less burnout (path $b$ coefficient $=-.29, p<.01)$. A bias-corrected bootstrap confidence interval for the indirect effect $(a b=-.13)$, based on 10,000 bootstrap samples, was entirely below zero $(95 \%$ CI: -.26 to -.03$)$. There was no evidence that self-compassion influenced burnout independent of its effect on the need for autonomy $\left(c^{\prime}=-.08, p=.15\right)$. In addition, there was no evidence that self-compassion indirectly influences burnout through either relatedness (95\% CI: -.10 to $.01, p=.27$ ) or competence $(95 \% \mathrm{CI}$ : -.13 to $.02, p=.27)$.

A different picture was found with concern for others as the predicting variable. The analysis showed that concern for others indirectly influenced burnout through its effect on relatedness. Participants with higher healthy concern scores had higher relatedness needs than participants with lower concern scores (path $d$ coefficient $=0.32, p<.001$ ). In addition, participants who had high relatedness needs reported less burnout (path $e$ coefficient $=$ $-0.25, p<.05$ ). A bias-corrected bootstrap confidence interval for the indirect effect (de $=-0.08$ ) based on 10,000 bootstrap samples was below zero $(95 \% \mathrm{CI}$ : -0.18 to -0.02$)$. Concern for others did not influence burnout independent of its effect on the need for relatedness $\left(f^{\prime}=-.08, p=.36\right)$. In addition, there was no evidence that concern for others indirectly influenced burnout through either autonomy (95\% CI: -.09 to $.03, p=.51)$ or competence (95\% CI: -.07 to $.01, p=.37$ ). Finally, both age and PCE were directly related to burnout.

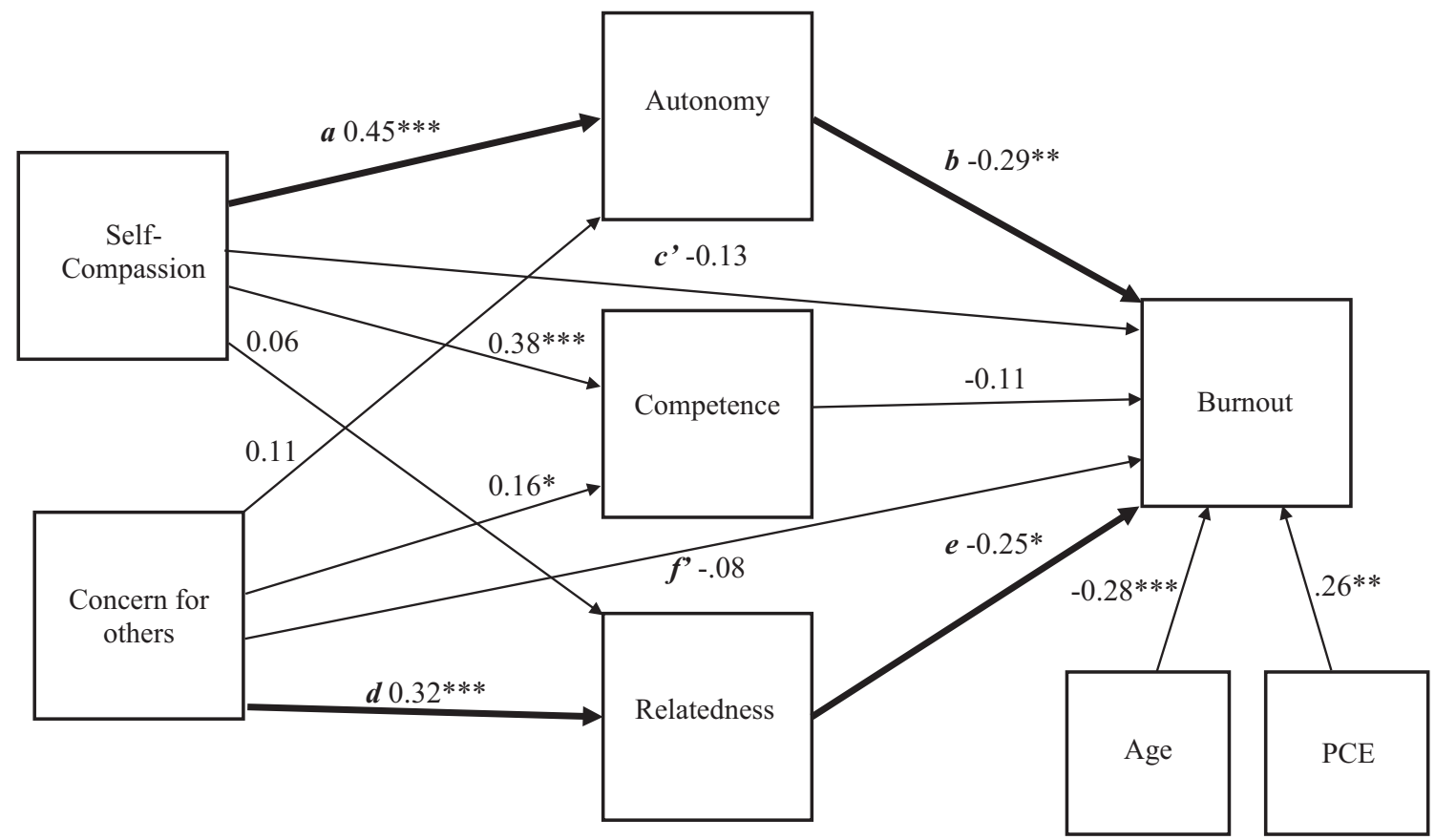

Fig. 1 Standardized coefficients of the PLS SEM path modeling. *** $p<0.001 ; * * p<0.01 ; * p<0.05$. PCE, particular clinical experience 


\section{Discussion}

The present study examined the association between selfcompassion, concern for others, and burnout among caregivers in a hospital setting. We fitted the collected data, based on multiple self-report measures, into a predetermined theoretical path model using a PLS-SEM approach. The findings show that different psychological self-needs mediate the relationship between self-compassion, concern for others, and caregiver's burnout. Specifically, autonomy mediated the relationship between self-compassion and burnout, while relatedness mediated the association between concern for others and burnout. The self-need of competence did not mediate any relationship between self-compassion, concern for others, and burnout. These results support our hypothesis that selfcompassion is associated with burnout, independent of concern for others, while concern for others is associated with burnout, regardless of self-compassion. Moreover, the simultaneous mediation model supports our theoretical presumptions of the mechanism by which self-compassion and concern for others complement each other, as illustrated in reduced burnout among caregivers.

Previous research has shown that both concern for others (i.e., compassion) and self-compassion are conducive to promoting wellbeing, such as alleviating psychological distress. For example, correlational studies have shown that greater self-compassion is associated with decreased depression and increased wellbeing (Gunnell et al. 2017; MacBeth and Gumley 2012; Neff 2011; Zessin et al. 2015). Although less definitive, similar findings were reported regarding concern for others (Arimitsu et al. 2019). Also, different intervention programs aimed at increasing either concern for others or selfcompassion have shown good outcomes (e.g., Barnard and Curry 2011; Kirby et al. 2017). In the present study, we focused on one aspect of wellbeing, namely occupational burnout. We posited that autonomy, a basic psychological selfneed in SDT, would mediate the unique covariance between self-compassion and burnout. According to Neff (2003a, b), self-compassion is comprised of three components: (a) mindfulness of the distressful situation, (b) self-kindness and nonjudgmental stance towards one's deeds, and (c) common humanity, which is seeing personal suffering as a universal human experience. Therefore, adopting a self-compassion approach is a way to accept one's failed actions and claim responsibility for their consequences without having to alienate oneself from them, distort, or deny them. We also reasoned that a self-compassionate individual, according to SDT, nourishes his need for autonomy. In the workplace, needs satisfaction is associated with reduced burnout (e.g., Bakker and Costa 2014; Deci et al. 2017). Thus, although a causal relationship cannot be determined between self-compassion, autonomy, and burnout, the theoretical ties that connect them appear to be probable.
Concern for others was found to be associated with burnout through the nutrient of relatedness. In other words, concern for others is a form of self-expression that nurtures the fundamental need for relatedness, which, in turn, reduces burnout. Relatedness is the sense of communal belonging felt when an individual expects to be cared for by others if necessary. Moreover, relatedness is also experienced when one gives or contributes to others (Deci and Ryan 2014). Thus, this sense of social attachment can be reached by either being the source or the target of the benevolent deed. The strong association between relatedness and burnout indicates that the former is an essential aspect of job satisfaction in the context of caregiving.

The combined association of self-compassion and concern for others with reduced burnout among caregivers indicates that self-compassion is a necessary complement to concern for others from an SDT perspective in the context of caregiving. Indeed, concern for others is an expression of one's exceptional human capacities in responding, being kind, and relating to others in need. However, as mentioned above, the practice of caregiving in a rehabilitative hospital setting is often grounded in a harsh reality involving irretrievable loss and partial recovery. Thus, caregivers must maintain a selfcompassionate nutriment of autonomy in determining the optimal course of action when their kindness and desire to heal are limited. The emotional strain inflicted on the caregivers by the subjects of their concern requires an ongoing and dynamic belief of the caregivers that their efforts are worthy and worthwhile. In sum, we understand self-compassion to be essential on behalf of the caregiver to persist, maintain satisfaction, and grow through his practice of caregiving over time. In organizational terms, it enables the caregiver to maintain the practice of ongoing control strategies as opposed to escapist strategies across all work scenarios, a primary beneficial coping strategy regarding burnout (Leiter 1991). The association of autonomy and relatedness with burnout, reflected in all the analyses performed (i.e., correlations, regression, and PLS-SEM path analysis), is relevant to the organizational psychology literature.

Competence, the third self-need, did not mediate the relationship between self-compassion, or concern for others, and burnout. According to SDT, competence reflects the individuals' need to feel that they can operate effectively in domains they believe they master. However, competence is easily thwarted when facing difficult unsolvable challenges and persistent vague and often negative feedback (Deci and Ryan 2014). In the present study, competence was related to self-compassion and concern for others but unrelated to burnout. This latter finding is surprising because one of burnout's components is inefficacy and the feeling of reduced accomplishment. One explanation for the lack of relationship between competence and burnout may be that certain job characteristics differently affect self-needs' satisfaction and, consequently, job burnout 
(Fernet et al. 2013). Thus, it is possible that the exhaustion and cynicism aspects of burnout had a more dominant role in the present sample of caregivers than inefficacy. However, in samples of other occupational settings, competence may be more salient (Babenko et al. 2018).

The current findings indicated that self-compassion and concern for others are unrelated. Moreover, different psychological needs mediated the covariance between these two constructs and burnout. These findings underscore previous findings regarding the independence of these two purportedly similar constructs (Gerber et al. 2015; López et al. 2018; Neff and Pommier 2013). In contrast, some research suggested that activation of support-giving schemas increased state self-compassion (Breines and Chen 2013). It is important to emphasize that this linkage was demonstrated mainly at the state level but not at the trait level. As research on compassion for self and others gains momentum, further clarifications of these concepts should be made. One approach to elucidate the nature of these affective tendencies is by assessing their relationship to other relevant constructs, as we did in the current study. Empirical differences and similarities will help set the theoretical boundaries between compassion for self and others and establish clearer definitions (Khoury 2019).

The theoretical model we presented here suggests how selfcompassion and concern for others are associated with burnout in caregiving professions through the mediating role of SDT's independent psychological needs. Recent studies have examined components of this model, and their findings are generally in line with ours. For example, Beaumont et al. (2016) reported that student midwives, reporting high levels of self-compassion and wellbeing, were less overwhelmed by compassion fatigue and burnout. Kotera et al. (2018) found that self-compassion and intrinsic motivation among UK caring profession students were associated with mental wellbeing and reduced psychological distress. Moreover, Babenko et al. (2018) found that, among Canadian medical students, selfcompassion and psychological needs were related to burnout-related exhaustion. Thus, although no study has examined the entire model presented in the current study, the incremental evidence of its different parts substantiates the entire theoretical edifice.

\section{Limitations and Future Research}

The present study has several limitations. First, the crosssectional design prevents us from making any causal inferences and undermines our proposed theoretical directionality. Future studies should manipulate pivotal variables, such as self-compassion and concern for others, and examine their (in)direct effects on burnout. The utilization of longitudinal designs may also strengthen the cause and effect relationships that our theory posits. Second, our research sample was considerably small, thereby limiting the clinical applicability of our results. Several methods exist for minimum sample size estimation in PLS-SEM, and future studies should adopt those that are more accurate such as the inverse square root method and the gamma-exponential method (Kock and Hadaya 2018). In addition, our sample consisted mostly of women, thus limiting its generalizability. The current mediation model's attempted replications should examine it in larger samples, particularly with a higher frequency of male participants. Moreover, the data collected was based on participants' self-reports, which are susceptible to confirmation and selfserving biases. Prospective investigations should devise ingenious, nonreactive measures that would circumvent these concerns. Finally, in the present study, we focused on the affective, empathic aspect of compassion as reflected in the measurement of concern for others. Future studies should also assess the association of motivational and cognitive factors of compassion with burnout among caregivers. Considering the apparent similarity between the concepts of compassion and self-compassion, the measurements of these constructs should share a similar as possible theoretical structure. Therefore, we recommend measuring compassion with the recently introduced compassion scale (CS) developed according to the same theoretical structure underlying the SCS (Khoury 2019; Pommier et al. 2020).

Contributions ZG designed and executed the study, assisted with the data analyses, and wrote the paper. DA collaborated with the design and writing of the study, analyzed the data, and wrote the results. Both authors collaborated in the writing and editing of the final manuscript.

\section{Compliance with Ethical Standards}

Conflict of Interest The authors declare that they have no conflicts of interest.

Ethical Approval The study was approved by the ethics committees of Reuth Rehabilitation Hospital (Tel Aviv, Israel) and of the Psychology Department at Bar-Ilan University (Ramat-Gan, Israel). Participants signed informed consent before participating in the study.

\section{References}

Allen, A. B., \& Leary, M. R. (2010). Self-Compassion, stress, and coping. Social and Personality Psychology Compass, 4, 107-118.

Arimitsu, K., Hitokoto, H., Kind, S., \& Hofmann, S. G. (2019). Differences in compassion, wellbeing, and social anxiety between Japan and the USA. Mindfulness, 10, 854-862.

Babenko, O., Mosewich, A., Abraham, J., \& Lai, H. (2018). Contributions of psychological needs, self-compassion, leisuretime exercise, and achievement goals to academic engagement and exhaustion in Canadian medical students. Journal of Educational Evaluation for Health Professions, 15, 1-7.

Bakker, A. B., \& Costa, P. L. (2014). Chronic job burnout and daily functioning: a theoretical analysis. Burnout Research, 1, 112-119. 
Barnard, L. K., \& Curry, J. F. (2011). Self-compassion: conceptualizations, correlates, \& interventions. Review of General Psychology, 15, 289-303.

Beaumont, E., Durkin, M., Martin, C. J. H., \& Carson, J. (2016). Compassion for others, self-compassion, quality of life and mental wellbeing measures and their association with compassion fatigue and burnout in student midwives: A quantitative survey. Midwifery, 34, 239-244.

Breines, J. G., \& Chen, S. (2013). Activating the inner caregiver: the role of support-giving schemas in increasing state self-compassion. Journal of Experimental Social Psychology, 49, 58-64.

Brown, K. W., \& Ryan, R. M. (2003). The benefits of being present: mindfulness and its role in psychological wellbeing. Journal of Personality and Social Psychology, 84, 822-848.

Davis, M. H. (1980). A multidimensional approach to individual differences in empathy. Catalog of Selected Documents in Psychology, 10, 1-17.

Deci, E. L., \& Ryan, R. M. (2000). The "what" and "why" of goal pursuits: human needs and the self-determination of behavior. Psychological Inquiry, 11, 227-268.

Deci, E. L., \& Ryan, R. M. (2014). Autonomy and need satisfaction in close relationships: Relationships motivation theory. In N. Weinstein (Ed.), Human motivation and interpersonal relationships: Theory, research, and applications (pp. 53-73). Dordrecht, Netherlands: Springer.

Deci, E. L., Olafsen, A. H., \& Ryan, R. M. (2017). Self-determination theory in work organizations: The state of a science. Annual Review of Organizational Psychology and Organizational Behavior, 4, 1943.

Faul, F., Erdfelder, E., Lang, A. G., \& Buchner, A. (2007). G* Power 3: A flexible statistical power analysis program for the social, behavioral, and biomedical sciences. Behavior Research Methods, 39, 175-191.

Fernet, C., Austin, S., Trépanier, S. G., \& Dussault, M. (2013). How do job characteristics contribute to burnout? Exploring the distinct mediating roles of perceived autonomy, competence, and relatedness. European Journal of Work and Organizational Psychology, 22, 123-137.

Finlay-Jones, A. L., Rees, C. S., \& Kane, R. T. (2015). Self-compassion, emotion regulation and stress among Australian psychologists: Testing an emotion regulation model of self-compassion using structural equation modeling. PloS One, 10, 1-19.

Gagné, M., \& Deci, E. L. (2005). Self-determination theory and work motivation. Journal of Organizational Behavior, 26, 331-362.

Gagné, M., \& Deci, E. L. (2014). The history of self-determination theory in psychology and management. In The Oxford handbook of work engagement, motivation, and self-determination theory. Oxford, UK: Oxford University Press.

Garson, G. D. (2016). Partial least squares. Regression and structural equation models. Statistical Publishing Associates.

Gerber, Z., \& Anaki, D. (2019). Self-compassion as a buffer against concrete but not abstract threat. Death studies, 43, 639-646.

Gerber, Z., Tolmacz, R., \& Doron, Y. (2015). Self-compassion and forms of concern for others. Personality and Individual Differences, 86, 394- 400 .

Gilbert, P. (2009). The compassionate mind. London, UK: Constable \& Robinson.

Goetz, J. L., Keltner, D., \& Simon-Thomas, E. (2010). Compassion: an evolutionary analysis and empirical review. Psychological Bulletin, 136, 351-374.

Gunnell, K., Mosewich, A., McEwen, C., Eklund, R., \& Crocker, P. (2017). Don't be so hard on yourself! Changes in self-compassion during the first year of university are associated with changes in wellbeing. Personality and Individual Differences, 107, 43-48.

Henseler, J., Hubona, G., \& Ray, P. A. (2016). Using PLS path modeling in new technology research: updated guidelines. Industrial Management \& Data Systems, 116, 2-20.
Hodgins, H. S., \& Knee, C. R. (2002). The integrating self and conscious experience. In E. L. Deci \& R. M. Ryan (Eds.), Handbook of selfdetermination research (pp. 87-100). Rochester: University of Rochester Press.

Hodgins, H. S., Weibust, K. S., Weinstein, N., Shiffman, S., Miller, A., Coombs, G., \& Adair, K. C. (2010). The cost of self-protection: Threat response and performance as a function of autonomous and controlled motivations. Personality and Social Psychology Bulletin, 36, 1101-1114.

Kanov, J. M., Maitlis, S., Worline, M. C., Dutton, J. E., Frost, P. J., \& Lilius, J. M. (2004). Compassion in organizational life. American Behavioral Scientist, 47, 808-827.

Khoury, B. (2019). Compassion: embodied and embedded. Mindfulness, 10, 2363-2374.

Kirby, J. N., Tellegen, C. L., \& Steindl, S. R. (2017). A meta-analysis of compassion-based interventions: current state of knowledge and future directions. Behavior Therapy, 48, 778-792.

Kock, N., \& Hadaya, P. (2018). Minimum sample size estimation in PLSSEM: The inverse square root and gamma-exponential methods. Information Systems Journal, 28, 227-261.

Kotera, Y., Green, P., \& Van Gordon, W. (2018). Mental wellbeing of caring profession students: relationship with caregiver identity, selfcompassion, and intrinsic motivation. Mindfulness \& Compassion, 3, 7-30.

Lazarus, R. S. (1991). Emotion and adaptation. New York: Oxford University Press.

Legault, L., \& Inzlicht, M. (2013). Self-determination, self-regulation, and the brain: autonomy improves performance by enhancing neuroaffective responsiveness to self-regulation failure. Journal of Personality and Social Psychology, 105, 123-138.

Leiter, M. P. (1991). Coping patterns as predictors of burnout: The function of control and escapist coping patterns. Journal of Organizational behavior, 12, 123-144.

López, A., Sanderman, R., Ranchor, A. V., \& Schroevers, M. J. (2018). Compassion for others and self-compassion: levels, correlates, and relationship with psychological wellbeing. Mindfulness, 9, 325331.

MacBeth, A., \& Gumley, A. (2012). Exploring compassion: a metaanalysis of the association between self-compassion and psychopathology. Clinical Psychology Review, 32, 545-552.

Maslach, C. (1982). Burnout: the cost of caring. Prentice Hall: Englewood Cliffs.

Maslach, C., Jackson, S. E., \& Leiter, M. P. (1997). Maslach burnout inventory: third edition. In C. P. Zalaquett \& R. J. Wood (Eds.), Evaluating stress: a book of resources (pp. 191-218). Scarecrow Education.

Mills, J., Wand, T., \& Fraser, J. A. (2018). Examining self-care, selfcompassion and compassion for others: a cross-sectional survey of palliative care nurses and doctors. International Journal of Palliative Nursing, 24, 4-11.

Montero-Marin, J., Zubiaga, F., Cereceda, M., Demarzo, M. M. P., Trenc, P., \& Garcia-Campayo, J. (2016). Burnout subtypes and absence of self-compassion in primary healthcare professionals: a crosssectional study. PloS One, 11, 1-17.

Moss, M., Good, V. S., Gozal, D., Kleinpell, R., \& Sessler, C. N. (2016). An official critical care societies collaborative statement: burnout syndrome in critical care health care professionals: a call for action. American Journal of Critical Care, 25, 368-376.

Muris, P., \& Petrocchi, N. (2016). Protection or vulnerability? A metaanalysis of the relations between the positive and negative components of self-compassion and psychopathology. Clinical Psychology and Psychotherapy, 24, 373-383.

Muris, P., Otgaar, H., \& Petrocchi, N. (2016). Protection as the mirror image of psychopathology: further critical notes on the SelfCompassion Scale. Mindfulness, 7, 787-790. 
Neff, K. D. (2003a). Development and validation of a scale to measure self-compassion. Self and Identity, 2, 223-250.

Neff, K. D. (2003b). Self-compassion: an alternative conceptualization of a healthy attitude toward oneself. Self and Identity, 2, 85-102.

Neff, K. D., \& Pommier, E. (2013). The relationship between selfcompassion and other-focused concern among college undergraduates, community adults, and practicing meditators. Self and Identity, $12(2), 160-176$.

Neff, K. D. (2011). Self-compassion, self-esteem, and wellbeing. Social and Personality Psychology Compass, 5, 1-12.

Neff, K. D., Long, P., Knox, M., Davidson, O., Kuchar, A., Costigan, A., Williamson, Z., Rohleder, N., Tóth-Király, I., \& Breines, J. G. (2018). The forest and the trees: Examining the association of selfcompassion and its positive and negative components with psychological functioning. Self and Identity, 17, 627-645.

Neff, K. D., Tóth-Király, I., Yarnell, L. M., Arimitsu, K., Castilho, P., Ghorbani, N., Guo, H. X., Hirsch, J. K., Hupfeld, J., Hutz, C. S., Kotsou, I., Lee, W. K., Montero-Marin, J., Sirois, F. M., de Souza, L. K., Svendsen, J. L., Wilkinson, R. B., \& Mantzios, M. (2019). Examining the factor structure of the Self-Compassion Scale in 20 diverse samples: support for use of a total score and six subscale scores. Psychological Assessment, 31, 27-45.

Neff, K. D., Knox, M. C., Long, P., \& Gregory, K. (2020). Caring for others without losing yourself: An adaptation of the Mindful SelfCompassion Program for Healthcare Communities. Journal of Clinical Psychology, 76, 1543-1562.

Pommier, E., Neff, K. D., \& Tóth-Király, I. (2020). The development and validation of the Compassion Scale. Assessment, 27, 21-39.

Potter, P., Divanbeigi, J., Berger, J., Norris, L., \& Olsen, S. (2010). Compassion fatigue and burnout: prevalence among oncology nurses. Clinical Journal of Oncology Nursing, 14(5), 56-62.

Ringle, C. M., Wende, S., \& Becker, J.-M. (2015). SmartPLS 3. Bönningstedt: SmartPLS.

Ronen, S., \& Mikulincer, M. (2014). The foundation of autonomous motivation in the workplace: an attachment perspective. In M. Gagné (Ed.), Oxford handbook of work engagement, motivation, and self-determination theory (pp. 109-126). Oxford: Oxford University Press.

Ryan, R. M., Legate, N., Niemiec, C. P., \& Deci, E. L. (2012). Beyond illusions and defense: exploring the possibilities and limits of human autonomy and responsibility through self-determination theory. In P. R. Shaver \& M. Mikulincer (Eds.), Meaning, mortality, and choice (pp. 215-233). Washington, DC: American Psychological Association.
Ryan, R. M., \& Deci, E. L. (2017). Self-determination theory: Basic psychological needs in motivation, development, and wellness. Guilford Publications.

Ryan, R. M., \& Deci, E. L. (2000). The darker and brighter sides of human existence: Basic psychological needs as a unifying concept. Psychological Inquiry, 11(4), 319-338.

Schaufeli, W. B., Bakker, A. B., \& Van Rhenen, W. (2009). How changes in job demands and resources predict burnout, work engagement, and sickness absenteeism. Journal of Organizational Behavior, 30, 893-917.

Sheldon, K. M., \& Prentice, M. (2019). Self-determination theory as a foundation for personality researchers. Journal of Personality, $87(1), 5-14$.

Strauss, C., Taylor, B. L., Gu, J., Kuyken, W., Baer, R., Jones, F., \& Cavanagh, K. (2016). What is compassion and how can we measure it? A review of definitions and measures. Clinical Psychology Review, 47, 15-27.

Tolmacz, R. (2010). Forms of concern: a psychoanalytic perspective. In M. Mikulincer \& P. R. Shaver (Eds.), Prosocial motives, emotions, and behavior: The better angels of our nature (pp. 93-107). Washington, DC: American Psychological Association.

Trépanier, S. G., Fernet, C., \& Austin, S. (2013). Workplace bullying and psychological health at work: the mediating role of satisfaction of needs for autonomy, competence and relatedness. Work Stress, 27, $123-140$.

Walker, L. J. (2017). Compassion, burnout and self-care in NHS staff delivering psychological interventions (Unpublished doctoral dissertation). London: University of East London.

Woodruff, C. C., \& Stevens, L. (2018). Where caring for self and others lives in the brain, and how it can be enhanced and diminished: observations on the neuroscience of empathy, compassion, and self-compassion. In L. Stevens \& C. C. Woodruff (Eds.), The neuroscience of empathy, compassion, and self-compassion (pp. 285320). San Diego: Academic Press.

Ying, Y. W., \& Han, M. (2009). Stress and coping with a professional challenge in entering masters of social work students: the role of self-compassion. Journal of Religion \& Spirituality in Social Work: Social Thought, 28, 263-283.

Zessin, U., Dickhäuser, O., \& Garbade, S. (2015). The relationship between self-compassion and wellbeing: a meta-analysis. Applied Psychology: Health and Well-Being, 7, 340-364.

Publisher's Note Springer Nature remains neutral with regard to jurisdictional claims in published maps and institutional affiliations. 